\title{
Bioactive Glass Nanoparticles-Loaded Poly( $\varepsilon$-caprolactone) Nanofiber as Substrate for ARPE-19 Cells
}

\author{
Tadeu Henrique Lima, ${ }^{1}$ Gabriella Maria Fernandes-Cunha, \\ Carlos Eduardo de Matos Jensen, ${ }^{3}$ Rodrigo Lambert Oréfice, ${ }^{1}$ \\ Armando da Silva-Cunha Junior, ${ }^{2}$ Min Zhao, \\ Francine Behar-Cohen, ${ }^{4,5,6}$ and Gisele Rodrigues da Silva ${ }^{3}$
}

${ }^{1}$ Department of Metallurgical and Materials Engineering, Federal University of Minas Gerais, 31270-901 Belo Horizonte, MG, Brazil

${ }^{2}$ Faculty of Pharmacy, Federal University of Minas Gerais, 31270-901 Belo Horizonte, MG, Brazil

${ }^{3}$ School of Pharmacy, Federal University of São João del-Rei, 35501-296 Divinópolis, MG, Brazil

${ }^{4}$ INSERM, U872, Team 17, Centre de Recherche des Cordeliers, 75006 Paris, France

${ }^{5}$ Université René Descartes Sorbonne Paris Cité, 75006 Paris, France

${ }^{6}$ Assistance Publique Hôpitaux de Paris, Hôtel-Dieu de Paris, 75004 Paris, France

Correspondence should be addressed to Gisele Rodrigues da Silva; giselersilva@ufsj.edu.br

Received 19 April 2016; Revised 10 June 2016; Accepted 22 June 2016

Academic Editor: Andrea Falqui

Copyright (C) 2016 Tadeu Henrique Lima et al. This is an open access article distributed under the Creative Commons Attribution License, which permits unrestricted use, distribution, and reproduction in any medium, provided the original work is properly cited.

\begin{abstract}
Bioactive glass nanoparticles-loaded poly( $\varepsilon$-caprolactone) nanofibers (BIOG PCL nanofibers) were synthesized and evaluated as substrates for ocular cells (ARPE-19). BIOG PCL nanofibers were characterized using SEM, FTIR, and DSC, and the in vitro degradation profile was also investigated. The in vitro ocular biocompatibility of nanofibers was exploited in Müller glial cells (MIOM1 cells) and in chorioallantoic membrane (CAM); and the proliferative capacity, cytotoxicity, and functionality were evaluated. Finally, ARPE-19 cells were seeded onto BIOG PCL nanofibers and they were investigated as supports for in vitro cell adhesion and proliferation. SEM images revealed the incorporation of BIOG nanoparticles into PCL nanofibers. Nanoparticles did not induce modifications in the chemical structure and semicrystalline nature of PCL in the nanofiber, as shown by FTIR and DSC. MIO-M1 cells exposed to BIOG PCL nanofibers showed viability, and they were able to proliferate and to express GFAP, indicating cellular functionality. Moreover, nanofibers were well tolerated by CAM. These findings suggested the in vitro ocular biocompatibility and absence of toxicity of these nanofibers. Finally, the BIOG nanoparticles modulated the protein adsorption, and, subsequently, ARPE-19 cells adhered and proliferated onto the nanostructured supports, establishing cell-substrate interactions. In conclusion, the biodegradable and biocompatible BIOG PCL nanofibers supported the ARPE-19 cells.
\end{abstract}

\section{Introduction}

The most widespread disease associated with retinal pigment epithelium (RPE) dysfunction is the age-related macular degeneration (AMD) [1,2]. The AMD is classified into two subgroups: atrophic (dry form) and exudative (wet form). The dry form is characterized by a progressive degeneration of RPE and photoreceptors. The exudative form is linked to choroidal neovascularization directed toward the subretinal macular region, with subsequent bleeding and/or fluid leakage, causing damage to the RPE layer, which may result in a sudden central vision loss $[2,3]$.

The treatment of AMD is based on systemic intravenous injections and intravitreal injections of drugs. The latter is the most effective technique to deliver therapeutic agents directly into the vitreous cavity, near the site of action. However, intravitreal injections are highly invasive and have many limitations, including patient noncompliance issues and risk of vitreous hemorrhage, retinal detachment, and development of cataracts [4-7]. Thus, new strategies have 
been investigated to overcome the drawbacks of conventional therapy of AMD.

One alternative to restrict the visual deterioration in AMD patients is RPE transplants. The RPE monolayer needs to be supported by a substrate and subsequently transplanted to repopulate Bruch's membrane. This substrate protects the RPE monolayer from the diseased Bruch's membrane, which prevents cell adhesion and proliferation [1]. Many biomaterials have been evaluated as substrate for RPE transplantation, including gelatin [8, 9], biodegradable polyurethanes [2], parylene-C $[10,11]$, plasma polymers of acrylic acid, allyl amine, and allyl alcohol [12].

Polymeric nanofibers have also been evaluated not only as vehicles for RPE transplantation, but also as Bruch's membrane substitutes. The three-dimensional (3D) morphology of nanometric fibers could mimic the natural fibrous architecture of human Bruch's membrane [13]. Additionally, their high porosity and pore interconnectivity, as well as large surface area-to-volume ratios, could enable efficient exchange of nutrients, gases, and waste products [14] for the adhered $\mathrm{RPE}$ cells in the in vivo microenvironment, thereby, ensuring the success of cell transplantation. In this context, ultrathin 3D nanofibrous membranes composed of different polymeric blends, collagen type I and (lactic-co-glycolic acid) (PLGA) [13], wild Antheraea pernyi silk fibroin, poly( $\varepsilon$-caprolactone), and gelatin [15], were designed for growth of human RPE cells and for potential use as prosthetic Bruch's membrane for RPE transplantation. Although these biomaterials mimic the human natural membrane and provide the adhesion and the proliferation of RPE cells, there are no approved substrates for cellular therapy in AMD patients.

In this study, electrospun nanofibers composed of poly $(\varepsilon-$ caprolactone) (PCL) hybridized with bioactive glass nanoparticles (BIOG) (BIOG PCL nanofibers) were synthesized and evaluated as a support for in vitro adhesion, proliferation, and uniform distribution of monolayer human RPE (ARPE19) cells. The PCL was selected to compose the BIOG PCL nanofibers due to its excellent spinnability, favorable mechanical properties, biocompatibility, and controllable biodegradation [16]. As the hydrophobicity of electrospun nanofibers composed of PCL limits the cell attachment [17], BIOG nanoparticles were inserted into the polymer solution, during the nanofiber manufacture process, to provide functionality to the nanostructured biomaterial. Spherical BIOG nanoparticles were prepared using the sol-gel process based on the reaction of alkoxides and other precursor salts in aqueous media using methanol as surfactant for obtaining oxideternary nanoparticles with the stoichiometric proportion of $60 \% \mathrm{SiO}_{2}, 36 \% \mathrm{CaO}$, and $4 \% \mathrm{P}_{2} \mathrm{O}_{5}$ [18].

The morphology of electrospun BIOG PCL nanofibers was demonstrated using scanning electron microscopy (SEM) and they were characterized by applying analytical techniques [Fourier Transform Infrared (FTIR) and Differential Scanning Calorimetry (DSC)]. The degradation profile of BIOG PCL nanofibers was also evaluated. The in vitro biocompatibility of nanofibers was demonstrated using the chorioallantoic membrane of hen's eggs and the parameters of tolerance. Additionally, the in vitro ocular biocompatibility was also analyzed using Müller glial cells (MIO-M1 cells), considering their viability and morphology in direct contact with BIOG PCL nanofibers. Finally, they were evaluated as support for in vitro attachment and proliferation of ARPE-19 cells. It was hypothesized that BIOG PCL nanofibers could be used as temporary substrate for cellular therapy for the treatment of AMD, which is associated with RPE dysfunction and progressive degeneration of photoreceptors.

\section{Materials and Methods}

2.1. Synthesis of Bioactive Glass Nanoparticles (BIOG Nanoparticles). BIOG nanoparticles were prepared by an alkalimediated sol-gel method and they were obtained in the ternary $\mathrm{SiO}_{2}-\mathrm{CaO}-\mathrm{P}_{2} \mathrm{O}_{5}$ system [19]. In brief, methanol (200 mL) (Sigma Chemical Co., USA), 33\% ammonium hydroxide solution $\left(\mathrm{NH}_{4} \mathrm{OH}, 0.46 \mathrm{~mL}\right.$ ) (Sigma Chemical Co., USA), and distilled water $(0.51 \mathrm{~mL})$ were stirred for 5 minutes. Then, 98\% tetraethyl orthosilicate solution (TEOS: $\mathrm{C}_{8} \mathrm{H}_{20} \mathrm{O}_{4} \mathrm{Si}, 5.57 \mathrm{~mL}$ ) (Sigma Chemical Co., USA), precursor of $\mathrm{SiO}_{2}$, and $99 \%$ triethyl phosphate (TEP: $\mathrm{C}_{6} \mathrm{H}_{15} \mathrm{O}_{4} \mathrm{P}$, $0.56 \mathrm{~mL}$ ) (Sigma Chemical Co., USA), precursor of $\mathrm{P}_{2} \mathrm{O}_{5}$, were added dropwise for 10 minutes. The molar ratio between water and TEOS was 1.86 . The sol was stirred for 48 hours and then placed in an oven at $50^{\circ} \mathrm{C}$ until the complete ammonium evaporation (approximately 3 hours). Then, it was filtered in a $0.22 \mu \mathrm{m}$ filter (Merck Millipore, Germany). The calcium nitrate tetrahydrate $\left(\mathrm{Ca}\left(\mathrm{NO}_{3}\right)_{2} \cdot 4 \mathrm{H}_{2} \mathrm{O}, 3.46 \mathrm{~g}\right)$ (Synth, Brazil) was dissolved in the sol and mixed for 24 hours. The developed nanoparticles were separated by subsequent filtrations in $0.22 \mu \mathrm{m}$ and $0.11 \mu \mathrm{m}$ filters (Merck Millipore, Germany) and freeze-dried to prevent the aggregation of gel particles during the drying process [20]. The obtained powder was thermally treated at $200^{\circ} \mathrm{C}$ for 40 minutes, at a heating rate of $1^{\circ} \mathrm{C} /$ minute. At the end of process, welldispersed bioactive glass nanoparticles (BIOG nanoparticles) were obtained without grinding and sieving [18, 21]. These spherical nanoparticles were extensively characterized and showed composition of $60 \% \mathrm{SiO}_{2}, 36 \% \mathrm{CaO}$, and $4 \% \mathrm{P}_{2} \mathrm{O}_{5}$ (\% mass). The average particle diameter was $87 \pm 4 \mathrm{~nm}$ with the polydispersion index of $0.10 \pm 0.01$ and pore diameter equal to $9.7 \mathrm{~nm}$. These nanoparticles exhibited a mesoporous structure attributed to their large surface area. They showed dispersion capability in water [18].

\subsection{Preparation of Bioactive Glass Nanoparticles-Loaded} Poly( $\varepsilon$-caprolactone) Nanofibers (BIOG PCL Nanofibers). The poly( $\varepsilon$-caprolactone) (PCL, MW 80,000-90,000 g/mol; Sigma Chemical Co., USA) solution $[14.6 \%(\mathrm{w} / \mathrm{v})]$ was prepared by dissolving PCL pellets in a mixture of acetic acid and formic acid (1:1) (Sigma-Aldrich, St. Louis, MO), under magnetic stirring for 3 hours at room temperature. The BIOG nanoparticles were added to the polymeric solution $[1 \%(\mathrm{w} / \mathrm{v})]$, under magnetic stirring for 1 hour at room temperature. The solution was electrospun using an electrospinning setup consisting of a dual polarity, high-voltage DC power supply unit (Gamma High Voltage Research, Ormond Beach, FL), a syringe pump (Arti Glass, CE, Italy), syringe (Arti Glass, CE, Italy), and a needle $(22 \mathrm{G})$ with blunted tip. The positive terminal of the high-voltage supply was 
connected to the needle tip while the negative terminal was connected to a metallic collector plate; a voltage of $+25 \mathrm{kV}$ was maintained between them. The flow rate was maintained at $3.0 \mathrm{~mL} / \mathrm{h}$ and the needle tip to collector distance was maintained at $10 \mathrm{~cm}$. The temperature and humidity inside the equipment were $25^{\circ} \mathrm{C}$ and $65 \%$, respectively. Bioactive glass nanoparticles-loaded poly( $\varepsilon$-caprolactone) nanofibers (BIOG PCL nanofibers) and PCL nanofibers (without BIOG nanoparticles) were collected on coverslips kept over the metallic collector disc ( $8 \mathrm{~cm}$ of diameter).

\subsection{Characterization}

2.3.1. Morphology and Diameter of Nanofibers. Morphology and diameter of BIOG PCL nanofibers and PCL nanofibers were analyzed by scanning electron microscopy (SEM) (JEOL JSM 5600, Japan) at an accelerating voltage of $15 \mathrm{kV}$. Samples for SEM were mounted on metal stubs and coated with gold using a sputter coater (JEOL JFC-1200 fine coater, Japan). Nonwoven nanofiber mats were analyzed with 50 individual measurements of nanofiber diameters taken from SEM micrographs using image analysis software (Image J, National Institutes of Health, USA). This was repeated for a single electrospun fiber mat fabricated under a single set of constant conditions to calculate the average nanofiber diameter and standard deviation [22].

2.3.2. Fourier Transform Infrared (FTIR). Infrared spectra were collected in a FTIR spectrophotometer (Perkin Elmer, model Spectrum 1000). Measurements were carried out using the attenuated total reflectance (ATR) technique. Each spectrum was a result of 32 scans with a resolution of $4 \mathrm{~cm}^{-1}$ [23].

2.3.3. Differential Scanning Calorimetry (DSC). Differential Scanning Calorimetry (DSC) curves were obtained on a Mettler Toledo DSC (Switzerland). Samples were put into aluminum pans. The calorimeter was calibrated for temperature and heat flow accuracy using pure indium melting (m.p. of $156.6^{\circ} \mathrm{C}$ and $\Delta \mathrm{H}=25.45 \mathrm{Jg}^{-1}$ ). The temperature ranged from 0 to $180^{\circ} \mathrm{C}$ with a heating rate of $25^{\circ} \mathrm{C} \mathrm{min}{ }^{-1}$ under nitrogen atmosphere [23].

2.3.4. Measurement of Weight Loss of BIOG PCL Nanofibers and PCL Nanofibers. The in vitro degradation study was evaluated by recording the weight loss of BIOG PCL nanofibers and PCL nanofibers over 15 days in simulated body fluid (SBF, pH of 7.4) [24]. Samples were placed in different tubes containing $3 \mathrm{~mL}$ of SBF ( $n=5$ for each sample). Those tubes were placed inside an incubator set at $37^{\circ} \mathrm{C}$ and $30 \mathrm{rpm}$. At each time-point (1, 2, 5, 10, and 15 days), samples were retrieved from the SBF, rinsed with deionized water, and vacuum-dried for $48 \mathrm{~h}$. The weight loss was analyzed. The percentage of weight loss was obtained by the ratio between the weight of samples before and after incubation.

\subsection{BIOG PCL Nanofibers as Substrates for ARPE-19 Cells}

2.4.1. ARPE-19 Culture. ARPE-19 cells, an established but nonimmortalized human RPE cell line, were grown in
Dulbecco's modified eagles medium and Ham's F12 medium (DMEM/F12, Gibco BRL: Grand Island, NY) with 10\% fetal bovine serum (FBS, Gibco BRL: Grand Island, NY) in a $37^{\circ} \mathrm{C}$ humidified atmosphere of $5 \% \mathrm{CO}_{2}$ and $95 \%$ air. The culture medium was refreshed every 2 days. Upon confluence, cells were rinsed with $2 \mathrm{~mL}$ of $0.05 \%$ trypsin-EDTA (Gibco, Grand Island, NY) solution and incubated with $5 \mathrm{~mL}$ of trypsin-EDTA at $37^{\circ} \mathrm{C}$ in a humidified atmosphere of $5 \%$ $\mathrm{CO}_{2}$ and $95 \%$ air. Next, within 5-15 minutes, the trypsin enzyme activity was stopped by the addition of $5 \mathrm{~mL}$ of complete growth medium and centrifuged for 5 minutes at $1500 \mathrm{rpm}$. The supernatant was discarded, while the cells were resuspended in $13 \mathrm{~mL}$ of fresh medium and seeded onto culture flasks for further propagation and subsequent passages [22].

2.4.2. ARPE-19 Cells on BIOG PCL Nanofibers and PCL Nanofibers. BIOG PCL nanofibers and PCL nanofibers were cut into round pieces ( $4.5 \mathrm{~mm}$ in diameter) and disinfected by exposure to UV light for 90 minutes on each side prior to cell culture. ARPE-19 cells were plated on top of each nanofiber disk and polyester tissue culture polystyrene (TCPS) (Costar, Cambridge, MA), as a control, at a density of $4 \times 10^{3}$ cells/well [22].

2.4.3. Number of Adherent ARPE-19 Cells on BIOG PCL Nanofibers and PCL Nanofibers. After 8 hours in culture, the medium was aspirated and the cells on BIOG PCL nanofibers, PCL nanofibers, and control TCPS were rinsed with phosphate-buffered saline (PBS pH of 7.4) and fixed in $4 \%$ paraformaldehyde (Synth, Brazil) for 15 minutes. Next, fixed cells were rinsed again with PBS for 5 minutes and immersed in PBS containing 0.3\% Triton X-100 (SigmaAldrich) for 15 minutes. After rising in PBS for 5 minutes, nuclei were stained with propidium iodide (Sigma-Aldrich, St. Louis, MO, USA) in PBS $(1: 100)$ for 10 minutes at room temperature. Finally, the cells were washed five times at 5minute intervals with PBS and one time with water, mounted in Gel Mount and viewed using a fluorescent microscope attached to a digital camera. Five fields were photographed per nanofiber sample and control TCPS (total of 15 fields per surface per time-point). Nuclei were counted for each field of view $\left(0.59 \mathrm{~mm}^{2}\right)$. The average number of nuclei on the control surface was set as $100 \%$, while the average number of nuclei \pm 1 standard deviation (SD) on BIOG PCL nanofiber and PCL nanofiber surfaces was obtained as a percentage of the control. Data were showed as a histogram [2].

2.4.4. ARPE-19 Cell Proliferation on BIOG PCL Nanofibers and PCL Nanofibers (Nuclear Count). After 1, 2, 5, and 10 days in culture, the medium was aspirated and the ARPE-19 cells on BIOG PCL nanofibers, PCL nanofibers, and control TCPS were submitted to the same procedure described for the attachment study. The average number of nuclei \pm 1 standard deviation (SD) per surface per time-point was showed as a histogram [2].

2.4.5. Cytotoxicity of ARPE-19 Cells on BIOG PCL Nanofibers and PCL Nanofibers. After 1, 2, 5, and 10 days in culture, 
the medium was aspirated and the ARPE-19 cells on BIOG PCL nanofibers, PCL nanofibers, and control TCPS were rinsed with PBS. ARPE-19 cells were incubated with $150 \mu \mathrm{L}$ of 3-[4,5-dimethylthiazol-2-yl]-2,5-diphenyltertrazolium bromide (MTT) $(1 \mathrm{mg} / \mathrm{mL}$ in PBS) (Sigma Chemical, Saint Louis, CO). After 3 hours of incubation, cells were lysed with $100 \mu \mathrm{L}$ of isopropanol and absorbance values were measured at $570 \mathrm{~nm}$ versus $630 \mathrm{~nm}$ using a microplate reader (BioRad, San Diego, CA). The mean absorbance on the control surface was set as $100 \%$, while the mean absorbance \pm standard deviation on nanofibers was obtained as a percentage of the control. Data were showed as a histogram [22].

2.4.6. Morphology of ARPE-19 Cells: Immunofluorescence. At 10 days of culture, the ARPE-19 cells on BIOG PCL nanofibers and control TCPS were submitted to the same procedure described for the attachment study. After nuclei staining with DAPI, F-actin fibers were labelled with Phalloidin FITC (Sigma-Aldrich) in PBS $(1: 250)$ for 30 minutes at room temperature. Cells were then rinsed, mounted, and viewed using fluorescent microscope attached to a digital camera [22].

\subsection{In Vitro Biocompatibility Study}

2.5.1. Müller Glial Cell (MIO-M1 Cell) Culture. MIO-M1 cells were grown in Dulbecco's modified eagles medium/glutamax (DMEM/glutamax Gibco BRL: Grand Island, NY) with 10\% FBS (Gibco BRL: Grand Island, NY), 0.4\% gentamicin, and $0.1 \%$ amphotericin $\mathrm{B}$ at $37^{\circ} \mathrm{C}$ in a humidified atmosphere of $5 \% \mathrm{CO}_{2}$ and $95 \%$ air. The passages were performed as described for ARPE-19 culture [23].

2.5.2. MIO-M1 Cell Proliferation in Contact with BIOG PCL Nanofibers and PCL Nanofibers (Nuclear Count). After 1, 2, 5 , and 10 days in culture, the medium was aspirated and MIO-M1 cells in contact with BIOG PCL nanofibers, PCL nanofibers, and control TCPS were submitted for the same procedure described for the attachment study. The average number of nuclei \pm 1 standard deviation (SD) per surface per time-point was showed as a histogram.

2.5.3. Cytotoxicity of MIO-M1 Cells in Contact with BIOG PCL Nanofibers and PCL Nanofibers. After 1, 2, 5, and 10 days in culture, the medium was aspirated and the MIO-M1 cells in contact with BIOG PCL nanofibers, PCL nanofibers, and control TCPS were submitted to the same procedure described for the cytotoxicity study of ARPE-19 cells. The mean absorbance on the control surface was set as $100 \%$, while the mean absorbance \pm standard deviation on nanofibers was obtained as a percentage of the control. Data were showed as a histogram.

2.5.4. Morphology of the MIO-M1 Cells: Immunofluorescence. At 10 days of culture, the MIO-M1 cells in contact with BIOG PCL nanofibers and control TCPS were submitted to the same procedure described for the morphology of ARPE-19 cells.

At 10 days of culture, the MIO-M1 cells grown in contact with BIOG PCL nanofibers and control TCPS were fixed with
$4 \%(\mathrm{v} / \mathrm{v})$ paraformaldehyde for 30 minutes at room temperature, for the labelling of glial fibrillary acidic protein (GFAP). Fixed cells were incubated with PBS containing Triton X-100 $0.1 \%(\mathrm{v} / \mathrm{v})$ for 30 minutes. This was followed by incubation with polyclonal rabbit antibody against GFAP (1:100) (Dako, Trappes, France) at room temperature for 3 hours. After washing with PBS, an Alexa Fluor 488-conjugated goat antirabbit IgG (1:100) (Molecular Probes, Leiden, Netherlands) was applied for 60 minutes in the dark. Finally, nuclei were stained with DAPI (Sigma-Aldrich) in PBS (1:1250) for 5 minutes at room temperature. Then, cells were rinsed five times, mounted, and viewed using fluorescent microscope attached to a digital camera (Olympus DP70) [23].

\subsubsection{Hen's Egg Test-Chorioallantoic Membrane (HET-CAM)} Irritation Test. Fertilized hen's eggs were purchased from a poultry farm (Alimentos Rivelli, Brazil). The collected hen's eggs were incubated at $37 \pm 0.5^{\circ} \mathrm{C}$ and $40 \% \pm 4 \%$ relative humidity for 10 days. Eggs were turned every day during incubation but were left in a horizontal position for several minutes to assure that the embryo was properly positioned. On day 10, each egg was opened by cracking the underside of the egg against the edge of a plastic petri dish [25]. The CAM was exposed and samples were placed directly onto the CAM's surface. After 20 seconds, samples were discarded and the CAM was carefully washed with HEPES buffer $(\mathrm{pH}$ of 7.4) to ensure the total removal of the tested substance. The CAM was visually observed for 5 minutes $(0.5,2$, and 5 minutes) regarding the appearing of any of the following phenomena: hyperemia, hemorrhage, and coagulation for which a score was given [26]. Samples were as follows: PBS $\mathrm{pH}$ of 7.4 (negative control), $0.1 \mathrm{~mol} / \mathrm{L}$ sodium hydroxide solution (positive control), BIOG PCL nanofibers, and PCL nanofibers.

2.6. Statistical Analysis. Results were expressed as mean \pm standard deviation. Data were tested for normality and investigated for statistical significance using Student's $t$-test or the one-way analysis of variance (ANOVA) when appropriate. A $p$ value of less than 0.05 was considered significant.

\section{Results and Discussion}

The AMD affects the macular region of the retina, resulting in loss of central vision. This process starts by the damage of RPE, a layer underneath the neurosensory region of the retina. Bruch's membrane separates the RPE and the choroid layer, which can thicken with age, thereby, slowing the transport of metabolites and, consequently, the formation of subretinal deposits (drusen) [7]. Additionally, the ageing impairs the ability of macrophages to efflux intracellular cholesterol. This substance accumulates into these cells, resulting in a phenotype of monocyte that promotes vascular proliferation [7, 27]. As the AMD progresses, the vision loss occurs in one of two forms: dry AMD (geographic atrophy) or wet AMD (neovascular AMD due to choroidal neovascularization). The angiogenesis in the RPE monolayer due to a break in Bruch's membrane, the bleeding from the 


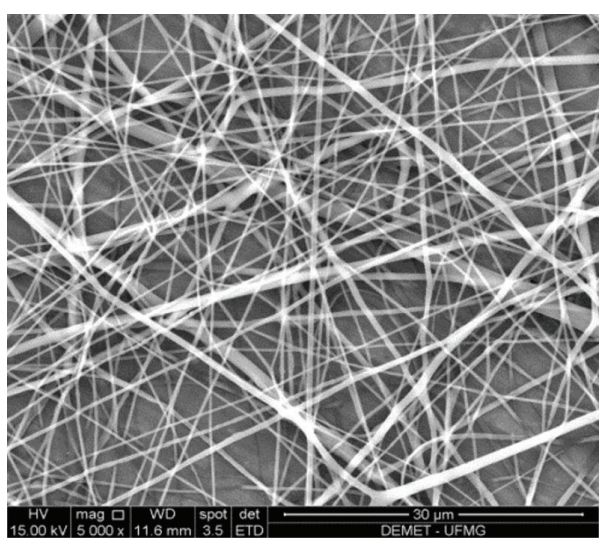

(a)

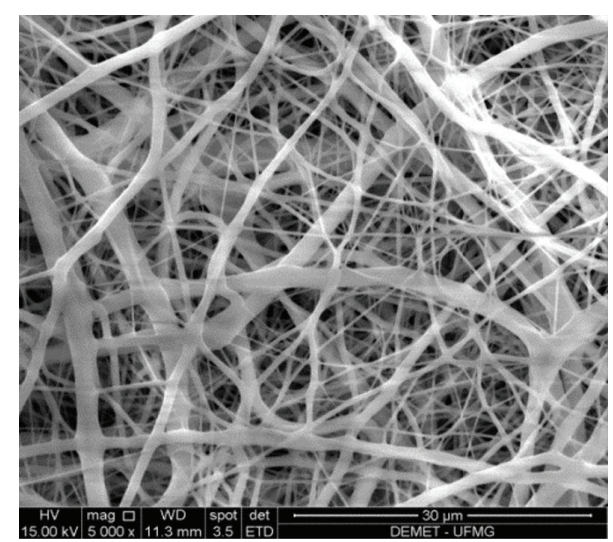

(b)

FIGURE 1: SEM images: morphology of electrospun PCL nanofibers (a) and BIOG PCL nanofibers (b). 5000x magnification. Scale bar: $30 \mu \mathrm{m}$.

vessels, the leakage of fluids, and the drusen lead to a rapid central vision loss $[4,7]$.

The regeneration of a diseased retina by transplantation of RPE cells represents an alternative for the treatment of AMD. The degenerated Bruch's membrane avoids the RPE growth and reconstitution. Therefore, a substrate needs to be applied as a support for RPE attachment and proliferation. This substrate may represent an artificial Bruch's membrane, containing a healthy RPE monolayer to be implanted into the subretinal space to cure blindness in degenerated macula.

In this study, BIOG PCL nanofibers were applied as substrates for ARPE-19 attachment and proliferation, aiming for the treatment of AMD. BIOG PCL nanofibers were produced by electrospinning the PCL with BIOG nanoparticles. The operational conditions (applied electric field of $+25 / 0 \mathrm{kV}$; needle to collector distance equivalent to $10 \mathrm{~cm}$; flow rate of $3 \mathrm{~mL} / \mathrm{h}$ ) and the PCL dissolution and BIOG nanoparticle dispersion in a mixture of acetic acid and formic acid $(1: 1)$ provided the formation of ultrafine polymeric fibers hybridized with BIOG nanoparticles. The high dielectric constant of the formic acid $(\varepsilon=57.5)$ along with the polyelectrolyte character of the formic acid and acetic acid was responsible for a high electrical conductivity, leading to a greater tensile force, which could increase the stretching and splitting of the jet resulting in a thin fiber [28].

The morphology of electrospun PCL nanofibers (Figure $1(\mathrm{a})$ ) and BIOG PCL nanofibers (Figure $1(\mathrm{~b})$ ) is shown in SEM images. The PCL nanofibers showed a complex network of fibers with a diameter of $109 \pm 10 \mathrm{~nm}$. The BIOG PCL nanofibers presented a $3 \mathrm{D}$ network of random oriented fibers embedded with the nanoparticles. They were internalized into the ultrafine fibers since the nanoparticles could not be individually visualized. The BIOG PCL nanofibers showed high porosity and diameter of approximately $344 \pm 45 \mathrm{~nm}$, which was superior to the diameter of PCL nanofibers. This fact was related not only to the incorporation of BIOG nanoparticles, but also to the increased viscosity of the polymeric solution in the presence of nanoparticles. This phenomenon led to the formation of thicker fibers during the electrospinning technique. Accordingly [29], an increase in the viscosity of spinning solution led to an increased nanofiber diameter. Moreover, the BIOG PCL nanofibers showed a rough surface compared to the surface of PCL nanofibers. The addition of $1 \%(\mathrm{w} / \mathrm{v})$ of bioglass yielded a structure with nanoparticle protuberances on the fiber surface. Lin and coworkers [24] demonstrated a similar phenomenon. In this study, PCL nanofibers containing BIOG nanoparticles were produced using the electrospinning technique, and the nanoparticles were attached to the polymeric fiber, producing a rough surface. The material surface roughness is an important factor for cell adhesion, proliferation, and differentiation $[24,30]$. Therefore, the cellular behavior can be influenced by the material surface in vitro.

The BIOG PCL nanofibers were characterized using different analytical techniques. Figure 2 showed the FTIR spectra of PCL nanofibers (Figure 2(a)), BIOG nanoparticles (Figure 2(b)), and BIOG PCL nanofibers (Figure 2(c)). The FTIR spectrum of PCL nanofibers (Figure 2(a)) showed typical bands of the polymer, such as between $2800 \mathrm{~cm}^{-1}$ and $3000 \mathrm{~cm}^{-1}$, due to symmetric and asymmetric $\mathrm{CH}_{2}$ stretching vibrations at approximately $1720 \mathrm{~cm}^{-1}$ corresponding to the $\mathrm{C}=\mathrm{O}$ stretching vibration, at $1290 \mathrm{~cm}^{-1}$ related to the $\mathrm{C}-\mathrm{C}$ and C-O stretching modes in the semicrystalline PCL [31], and at approximately $1160 \mathrm{~cm}^{-1}$ and at $1250 \mathrm{~cm}^{-1}$ equivalent to symmetric and asymmetric $\mathrm{C}-\mathrm{O}-\mathrm{C}$ stretching vibrations, respectively. The infrared results obtained from PCL nanofibers were similar to those previously described [32]. The FTIR spectra of BIOG nanoparticles (Figure 2(b)) demonstrated a broad band between $1200 \mathrm{~cm}^{-1}$ and $900 \mathrm{~cm}^{-1}$ associated with the $\mathrm{Si}-\mathrm{O}$ symmetric stretching of bridging oxygen atoms between tetrahedrons, $\mathrm{Si}-\mathrm{O}$ stretching of nonbridging oxygen atoms, and $\mathrm{Si}-\mathrm{O}-\mathrm{Si}$ symmetric and asymmetric stretching [33], corresponding to the silicate network. This broad band was overlapped with the infrared band corresponding to $\mathrm{P}-\mathrm{O}$ symmetric stretching vibration, which is normally detected at $1160 \mathrm{~cm}^{-1}$. The band at approximately $1500 \mathrm{~cm}^{-1}$ corresponded to the presence of organics, such as alkoxy groups [34]. The intensity of the infrared bands of BIOG nanoparticles is extremely low, demonstrating the existence 


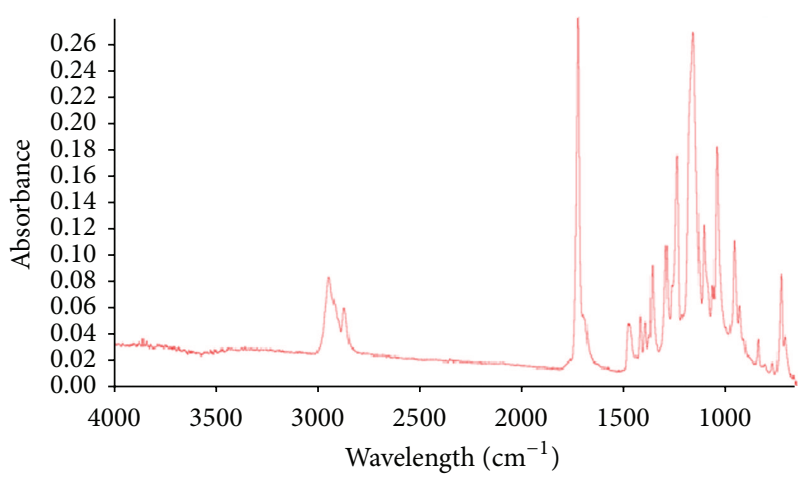

(a)

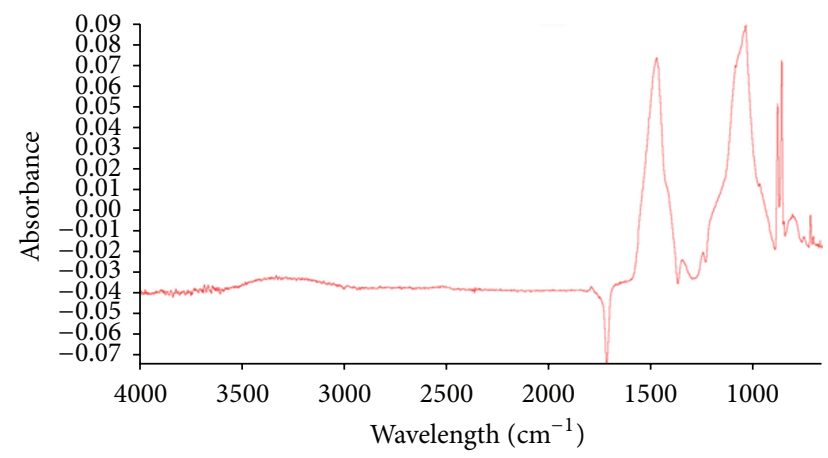

(b)

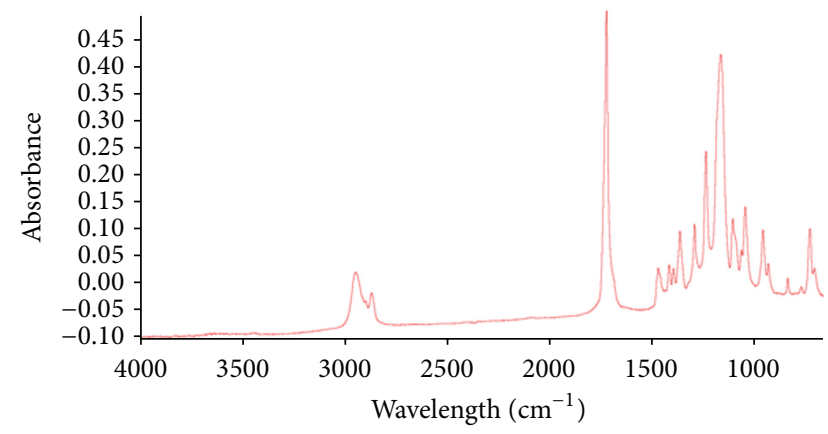

(c)

FIGURE 2: FTIR spectrum of PCL nanofibers (a), BIOG nanoparticles (b), and BIOG PCL nanofibers (c).

of an inorganic substance. Finally, the FTIR spectra of BIOG PCL nanofibers indicated that the bands attributed to the BIOG nanoparticles were completely superimposed by the bands of the polymer in nanofibers due to higher intensity of these polymeric bands constituted by organic groups. Additionally, the BIOG nanoparticles were not detected on the polymeric nanofibers by FTIR technique probably due to their lower proportion $[1 \%(\mathrm{w} / \mathrm{v})]$ related to the polymer. Poor evidence of substance peaks due to a small amount of this substance in different systems had been previously reported [35].

Figure 3 exhibited DSC curves of BIOG nanoparticles, PCL bulk, PCL nanofibers, and BIOG PCL nanofibers. DSC curves confirmed the semicrystalline nature of the PCL by showing the melting point temperature $(\mathrm{Tm})$ around $51^{\circ} \mathrm{C}$

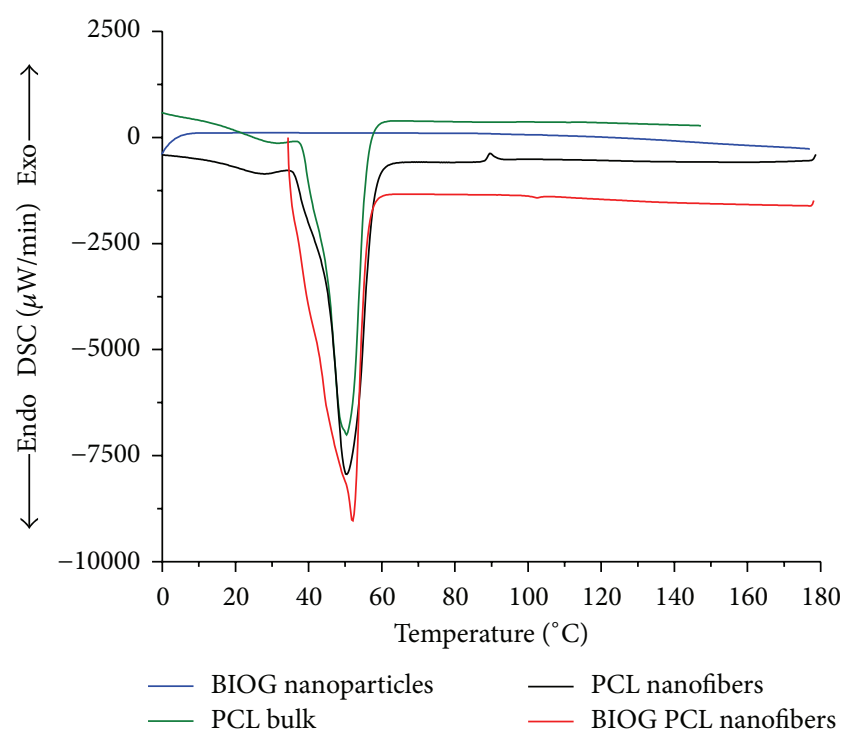

FIGURE 3: DSC curves of BIOG nanoparticles, PCL bulk, PCL nanofibers, and BIOG PCL nanofibers.

for all samples (PLC bulk, PCL nanofibers, and BIOG PCL nanofibers) [36, 37]. The incorporation of BIOG nanoparticles into the polymeric nanofibers did not affect significantly the semicrystalline nature of the PCL and the volume fraction of PCL crystals within the material (since both the melting point and enthalpy of melting are similar for all samples). Additionally, the electrospinning technique also did not drastically modify the volume fraction of crystals when nanofibers were compared to PCL bulk. Finally, thermal events of BIOG PCL nanoparticles were not observed until $180^{\circ} \mathrm{C}$ of heating probably due to the fact that nanoparticles were previously heat-treated at $200^{\circ} \mathrm{C}$.

The in vitro degradation of PCL nanofibers and BIOG PCL nanofibers was monitored by immersing them into the SBF ( $\mathrm{pH}$ of 7.4) for 15 days. The weight of nanofibers was measured and compared with the initial weight to indicate the percentage of the biomaterial that had been hydrolyzed. The PCL nanofibers showed progressive weight loss and only $5 \%$ of the initial weight of polymeric nanofibers remained after the period of 15 days of incubation (Figure 4). The degradation of PCL occurred due to the hydrolysis of ester bonds in polymeric chains, inducing an increase in the amount of carboxyl and hydroxyl groups in nanofibers. The $-\mathrm{COOH}$ groups ionized in the SBF $(\mathrm{pH}$ of 7.4) and associated with the - $\mathrm{OH}$ groups increased the hydrophilicity of the polymer. As a result, the water uptake increased, leading to the rapid degradation of PCL nanofibers and, consequently, their weight loss. Additionally, the water uptake also occurred due to the presence of pores in ultrafine fibers, conducting to the rapid degradation of polymeric nanofibers [38]. The BIOG PCL nanofibers showed an in vitro degradation profile different from that obtained for PCL nanofibers. At 2 days of incubation, the weight of these hybridized nanofibers increased; then it continuously decreased, and the biomaterial degraded completely after 


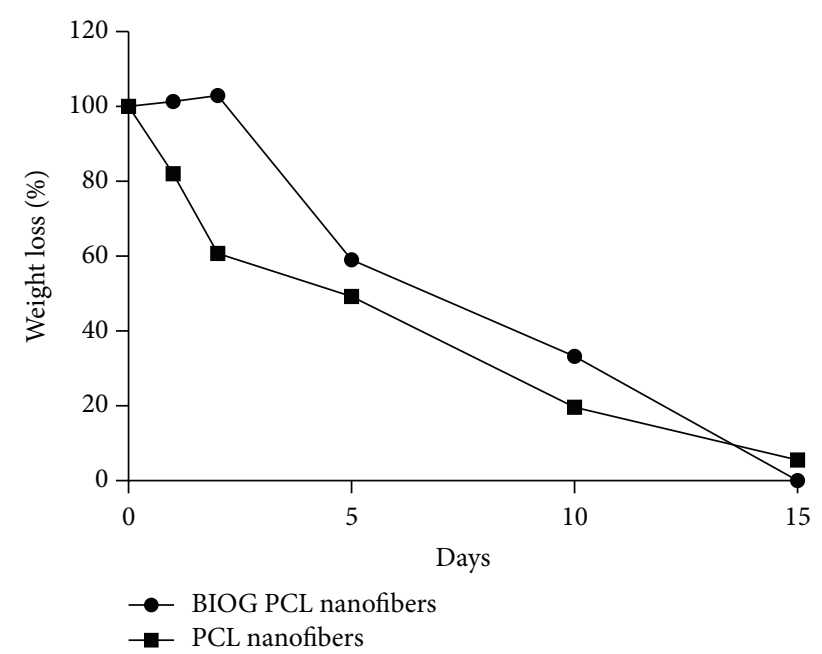

FIGURE 4: In vitro degradation profile of PCL nanofibers and BIOG PCL nanofibers during 15 days of incubation in SBF $(\mathrm{pH}, 7.4)(n=$ 5 for each sample). The standard deviation was lower than $5 \%$ for samples.

15 days of incubation (Figure 4). Immediately after BIOG PCL nanofibers were soaked in SBF, a sequence of interfacial reactions began, as previously proposed [24, 39]. There was an exchange of $\mathrm{H}_{3} \mathrm{O}^{+}$from SBF solution with $\mathrm{Ca}^{2+}$ on the surface of bioactive glasses, leading to the formation of silanol groups. Silanol groups supplied favorable sites for amorphous calcium phosphate nucleation that can evolve to crystalline calcium phosphates. Therefore, structural changes of bioactive glass in contact with SBF solution as well as the deposition of calcium phosphate species could be responsible for the increase in the nanofiber weight in the first 2 days of in vitro degradation test. The incorporation of bioactive glass nanoparticles into PCL nanofibers led to the formation of a bioactive composite. After this initial weight gain, the degradation of PCL in nanofibers became faster than the formation of apatite on the BIOG surface, and the scission of the polymeric chains occurred, leading to the progressive degradation of hybridized nanofibers in the next days of incubation.

The adhesion of ARPE-19 cells on the surfaces of BIOG PCL nanofibers, PCL nanofibers, and control TCPS was expressed as the mean number of cell nuclei (\%) \pm SD after 8 hours of incubation (Figure 5). The attachment rate on BIOG PCL nanofibers and PCL nanofibers was $86.54 \%$ and $9.23 \%$, respectively. The statistical analysis (Student's $t$-test) demonstrated that there was a difference in the mean number of cells attached to the nanofibers after 8 hours of incubation $(p>0.05)$. Therefore, the experimental attachment study indicated that ARPE-19 cells adhered better to BIOG PCL nanofibers than PCL nanofibers. The incorporation of BIOG nanoparticles into PCL nanofibers provided the bioactivity for these polymeric membranes, which probably induced the adsorption of serum adhesion proteins on the surfaces. These adsorbed proteins led to the cellular-substrate interactions and, consequently, the ARPE-19 cell attachment. Furthermore, the topography of hybridized nanofibers also induced

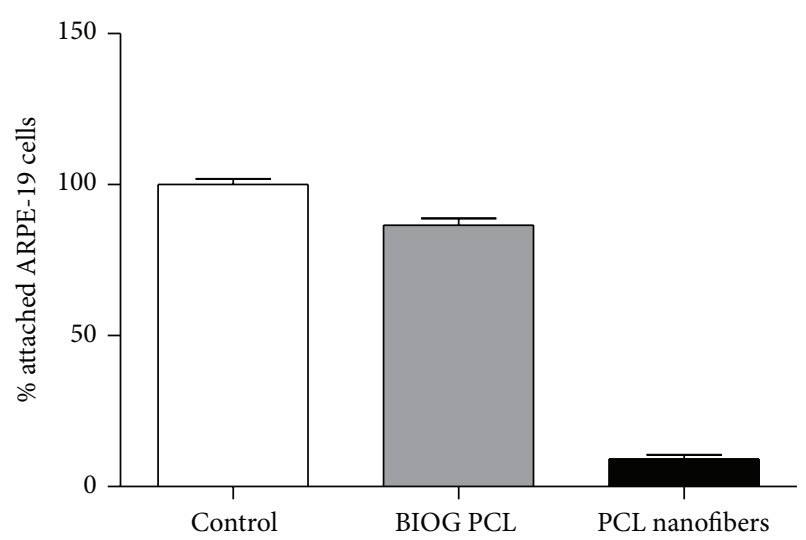

FIGURE 5: ARPE-19 cells attached to BIOG PCL nanofibers and PCL nanofibers after $8 \mathrm{~h}$ of seeding. Data were expressed as a percentage of the control TCPS \pm SD, fixed at $100 \%$ ( $n=15$ for each nanofiber and control) $(p>0.05)$.

the RPE adhesion since the amount of adsorbed proteins enhances due to the material surface roughness. This result corroborated with Rechendorff and coworkers [40], who demonstrated that the protuberances on material surface led to increased adsorbed proteins. On the other hand, the PCL nanofibrous matrix did not provide an expressive cellular adhesion and it could not be considered a substrate for ARPE19 cell attachment and proliferation. The hydrophobicity of PCL probably promoted repulsion of proteins and cells that approached the surface. The hydrophobic character of PCL represents a disadvantage of this polymer. However, its surface can be altered by blending PCL with other polymers or hybridizing with nanoparticles to make it more effective as cellular support [41].

The proliferation of ARPE-19 cells on the surface of BIOG PCL nanofibers, PCL nanofibers, and control TCPS was expressed as mean number of nuclei for each day of incubation (Figure 6(a)). Over the period of culture, the number of RPE nuclei increased significantly on the surface of BIOG PCL nanofibers and control TCPS, demonstrating cell proliferation. However, the proliferative capacity of ARPE19 cells was less on top of PCL nanofibers as compared to cells cultivated on BIOG PCL nanofibers and control (oneway ANOVA, $p>0.05$ ). The extracellular proteins, attached to the BIOG PCL surfaces, induced the adhesion of ARPE19 cells. These cells generated intracellular signs that affected cell spreading, growth, and proliferation ability. Therefore, the BIOG PCL nanofibers provided the establishment of cellpolymeric surface interactions once the RPE cells were able to attach, migrate, and proliferate on nanofibers [2].

Additionally, the ARPE-19 cells were cultured on top of BIOG PCL nanofibers, PCL nanofibers, and control TCPS to investigate the viability of these cells after 1,2 , 5, and 10 days of incubation (Figure 6(b)). The ARPE-19 cells showed viability of approximately $100 \%$, similar to the control. However, the viability of ARPE-19 cells was less on PCL nanofibers compared to the survival rate of cells cultivated on BIOG PCL nanofibers and control (one-way 


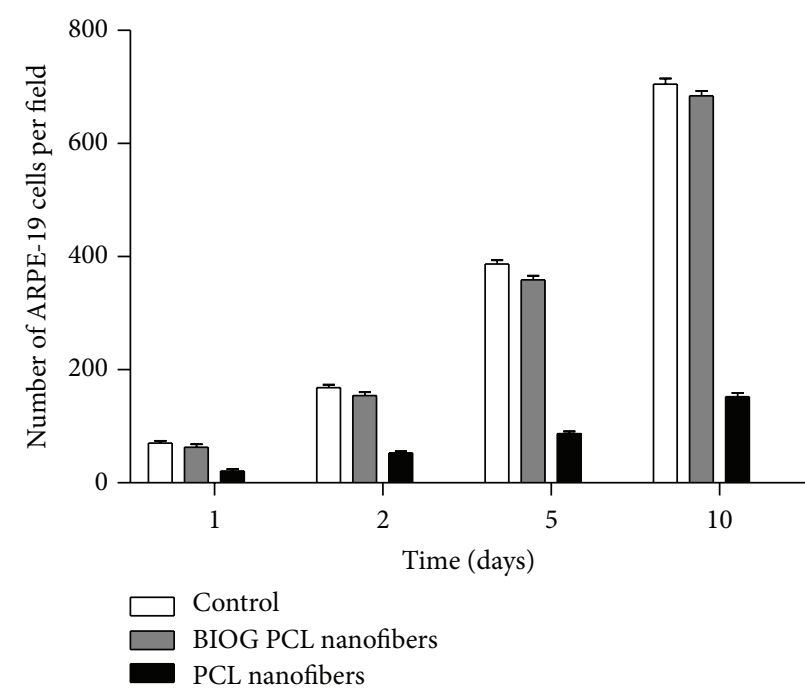

(a)

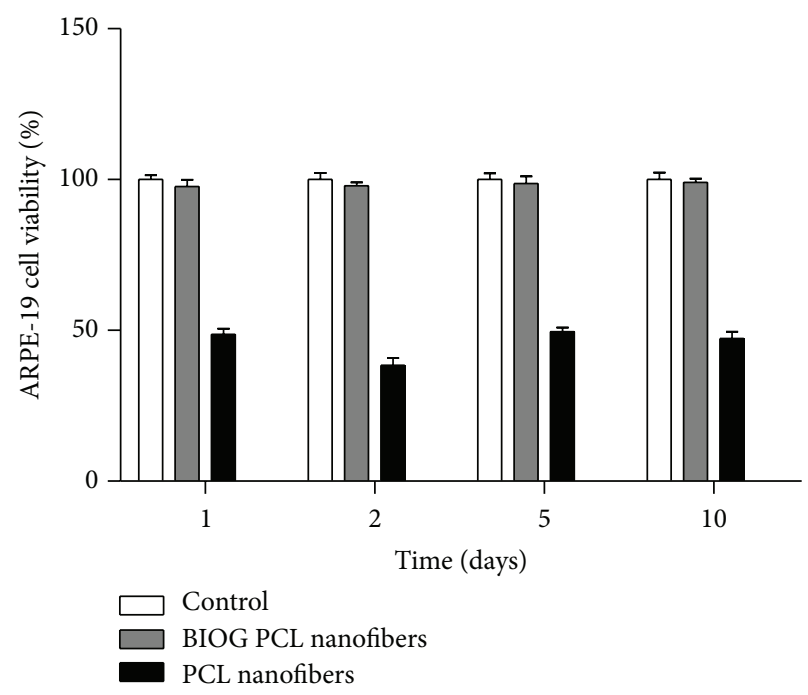

(b)

FIgURE 6: (a) Proliferation kinetics of ARPE-19 cells cultured in vitro on BIOG PCL nanofibers, PCL nanofibers, and control TCPS during 10 days. Data were expressed as mean number of nuclei \pm SD $(n=15$ for each nanofiber and control, per each day) $(p>0.05)$. (b) Viability of ARPE-19 cells cultured in vitro on BIOG PCL nanofibers, PCL nanofibers, and control TCPS during 10 days. Data were expressed as a percentage of the control TCPS \pm SD, fixed at $100 \%(n=15$ for each nanofiber and control $)(p>0.05)$.

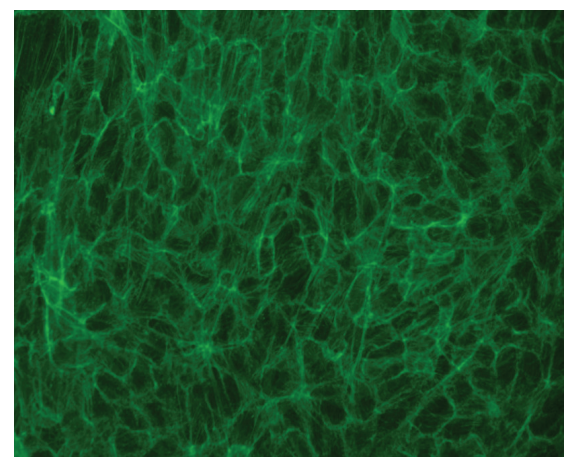

(a)

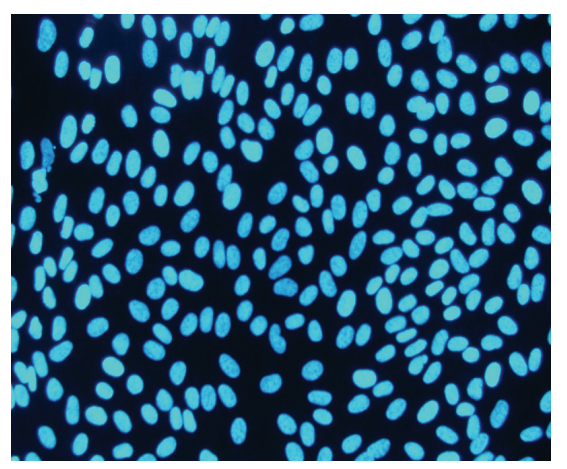

(b)

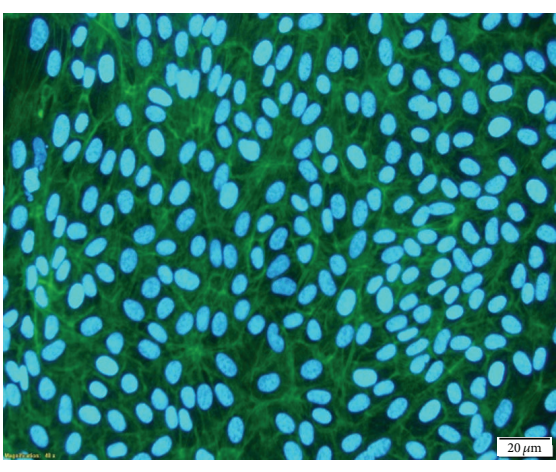

(c)

FIGURE 7: Micrographs of ARPE-19 cells cultured on BIOG PCL nanofiber surface after 10 days of incubation. The actin filaments were stained with FITC Phalloidin (green) (a); the nuclei were stained with DAPI (blue) (b); merge of stained F-actin and nuclei of cells (c). $(\times 40)$ scale bar: $20 \mu \mathrm{m}$.

ANOVA, $p>0.05)$. The cells on BIOG PCL nanofiber surface showed high survival rate over a 10-day period and they were metabolically active. This implied that the BIOG PCL nanofibers were suitable for supporting the growth of healthy cells. The existence of a porous structure in nanofibers offered optimum nutrient and gas exchanges for cell growth and survival [41]. As the cells retained their viability, the BIOG PCL nanofibers demonstrated biocompatibility and absence of toxicity. Furthermore, as previously shown, the ARPE-19 cells were not capable of adhering and proliferating on PCL nanofiber surfaces due to their high hydrophobicity, which prevented the adsorption of serum adhesion proteins and, consequently, cell-substrate interactions. Therefore, as the number of adhered cells on PCL nanofibers was decreased, the quantification of MTT was also decreased; consequently, a reduction in the viability was observed when compared to that in the control (fixed as 100\%).

The morphology of the ARPE-19 cells on BIOG PCL nanofiber surface and control TCPS was evaluated after 10 days of incubation (Figure 7). These cells reached confluence and demonstrated high density. The actin filaments, stained with Phalloidin, were disposed in the upper part of the cytoplasm and between adjacent cells, thus, providing a connection between them and representing large surface attachments [42]. The nuclei, stained with DAPI, did not overlap, indicating the existence of a single cell layer. The morphological assessment of ARPE-19 cells, described in this study, was comparable to the phenotype of RPE cells cultivated in vitro on top of a 3D ultrafine PLGA and collagen membrane, since they formed an organized monolayer of 


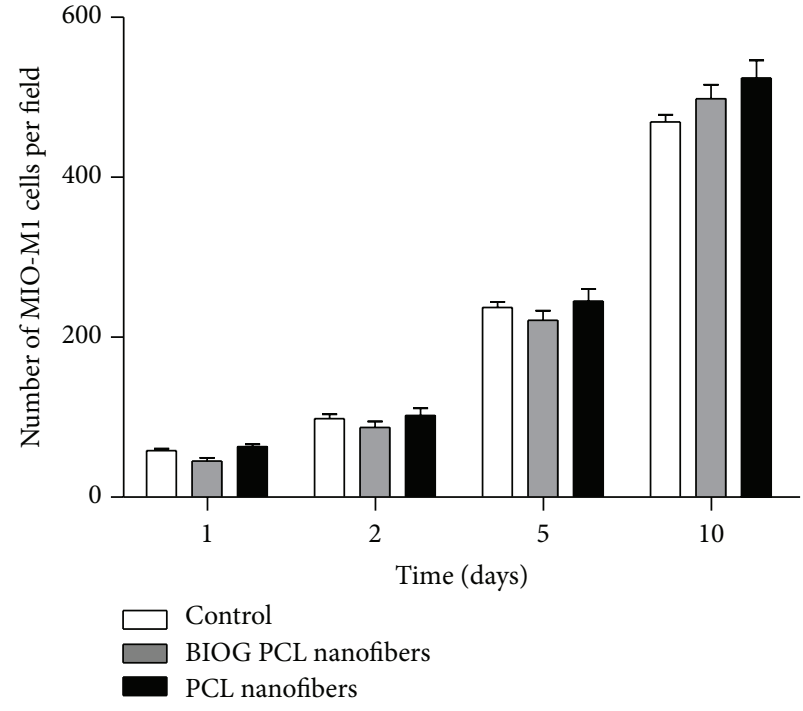

(a)

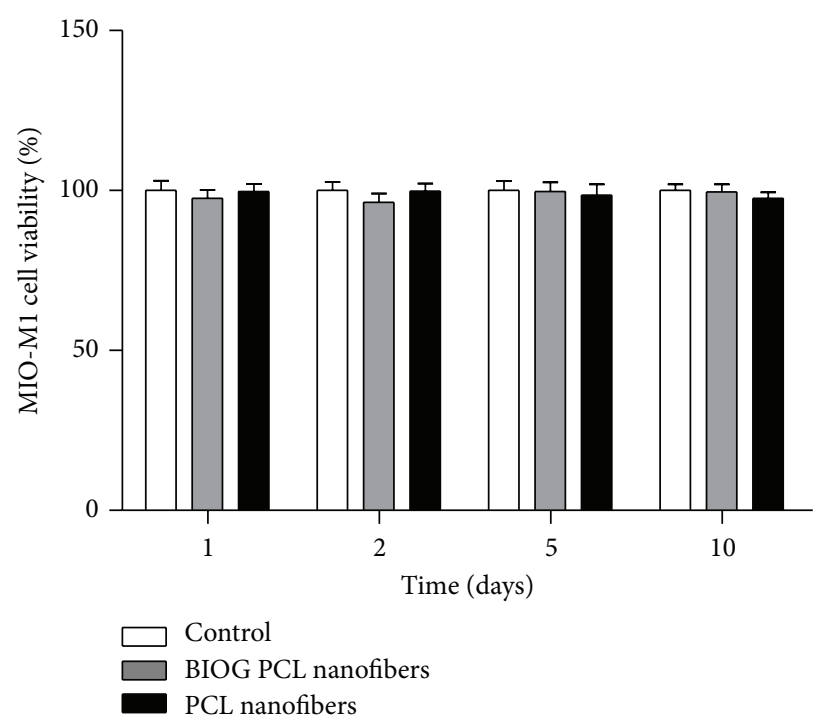

(b)

FIgURE 8: Proliferation kinetics (a) and viability (b) of MIO-M1 cells cultured in direct contact with BIOG PCL nanofibers, PCL nanofibers, and control TCPS at 1,2,5, and 10 days of incubation. The data of proliferation were expressed as mean number of nuclei \pm standard deviation for each time-point $(n=10$ per group per day) $(p<0.05)$. The viability was relative to the control, fixed at $100 \%$ ( $n=10$ per group per day) $(p<0.05)$.

highly interconnected cells [13]. The BIOG PCL nanofibers also presented a $3 \mathrm{D}$ network of random oriented fibers embedded with the nanoparticles. It was well documented that 3D nanofibers with their porous mesh-like fibrillar structures act as a biological sieve for reciprocal transfer of nutrients, biomolecules, oxygen, and metabolic waste, mimicking a healthy native Bruch's membrane and allowing the growth of functional RPE monolayer, which are essential to treat the AMD [13].

Finally, the BIOG PCL nanofibers showed protein absorbability, molecule transport, and ideal topography, which represented essential characteristics for adhesion, proliferation, and viability of ARPE-19 cells. These cells retained their native phenotype, supporting the formation of a cellular sheet. Furthermore, it is important to emphasize that the BIOG PCL nanofibers acted as temporary substrate for RPE cells, since these materials biodegraded due to the hydrolysis of ester bonds of PCL. The biodegradation of nanofibers is required for the establishment of the transplanted RPE cells in the damage ocular tissue.

Considering the possibility of applying the BIOG PCL nanofibers as substrates for ARPE-19 cells, these nanostructured materials may be indirectly in contact with the cells of the neurosensory retina when they are transplanted to the subretinal space. The existence of residues of organic solvents (acetic acid and formic acid) derived from the synthesis and entrapped in the fibers could induce an inflammatory response in the ocular tissues, damaging the neurosensory retina. Additionally, the possible presence of residues of nitrate by-products derived from the synthesis of BIOG nanoparticles could also promote a severe inflammatory reaction in the eye's tissues. The deterioration of these delicate structures would compromise the vision, leading to partial or complete vision loss. In this regard, further investigation of the ocular biocompatibility of BIOG PCL nanofibers must be performed. Then, studies on biocompatibility were conducted using Müller cells (MIO-M1 cells), which are an important part of neurosensory retinal layers.

The Müller cells are specialized glial cells, which span the entire thickness of the neural retina, from the vitread surface to the subretinal space. The processes of Müller cells contact the extracellular clefts around virtually each retinal neuron and ensheath neuronal somata and processes and blood vessels. The Müller cells support the functioning and metabolism of retinal neurons and they are active players in normal retinal functions as well as in virtually all types of retinal degeneration $[43,44]$.

The MIO-M1 cells were cultured in direct contact with BIOG PCL nanofibers, PCL nanofibers, and control TCPS to evaluate the proliferation of these glial cells after 1, 2, 5, and 10 days of incubation (Figure $8(\mathrm{a})$ ). The MIO-M1 cells were capable of proliferating in the presence of nanofibers and control. There was no statistical difference in the proliferation rate among these groups (one-way ANOVA, $p<0.05$ ). Moreover, the MIO-M1 cells were incubated in direct contact with BIOG PCL nanofibers, PCL nanofibers, and control TCPS to investigate their viability over a period of 10 days (Figure $8(\mathrm{~b})$ ). The viability of MIO-M1 cells incubated with the polymeric nanofibers was similar to the cell viability of the control. There was no significant statistical difference among these groups (one-way ANOVA, $p<0.05$ ). These findings suggested that the BIOG PCL nanofibers, the degradation products of the polymer, and the BIOG nanoparticles did not influence the growth and proliferative ability of these cells 


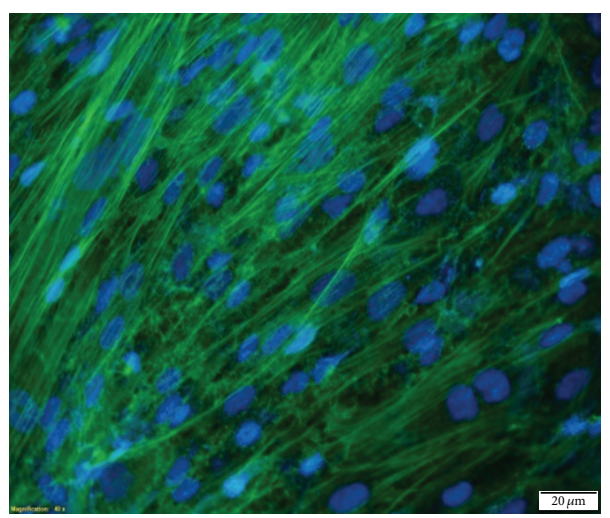

(a)

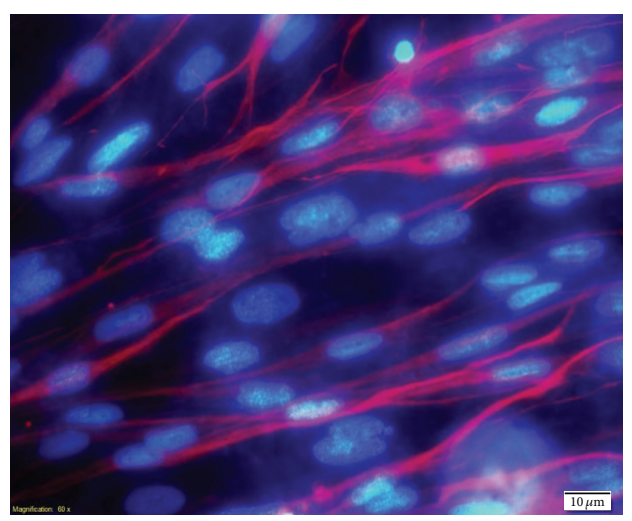

(b)

FIGURE 9: (a) Micrographs of MIO-M1 cells incubated in direct contact with BIOG PCL nanofibers for 10 days. The actin filaments were stained with FITC Phalloidin (green) and the nuclei were stained with DAPI (blue). ( $\times 40)$ scale bar: $20 \mu \mathrm{m}$. (b) GFAP (red) expressed by Müller glial cells. $(\times 60)$ scale bar: $10 \mu \mathrm{m}$.

in culture. They did not also influence the survival rate of cells, which demonstrated their metabolic activity. Therefore, the nanofibers, the by-products, and the nanoparticles were nontoxic to the MIO-M1 cells and showed in vitro ocular biocompatibility.

The morphology of MIO-M1 cells in the presence of BIOG PCL nanofibers and control was evaluated after 10 days of incubation (Figure 9(a)). These cells reached confluence and attained an elongate appearance, which was demarcated by actin filaments. This cell format was characteristic of radial glial cells [43]. Additionally, the ellipsoid nuclei were centrally located and they did not overlap, demonstrating the existence of a monolayer. A similar phenotype of MIO-M1 cells was previously reported [22]. In this work, the Müller cells were cultured in the presence of PCL nanofibers and these retinal cells expressed the microfilament actin, revealing the existence of elongated and radially oriented cells, and the ellipsoid nuclei were highly centralized. The MIO-M1 cells in contact with BIOG PCL nanofibers demonstrated their functionality, since they expressed the glial fibrillary acidic protein (GFAP) (Figure 9(b)). The GFAP is a ubiquitous marker for glia and an important cytoskeletal component determining cell morphology [45]. The production of GFAP indicated that cells were not under stress, since the upregulation of GFAP is an early event under retinal stress conditions [46]. Finally, the BIOG PCL nanofibers, the polymeric degradation products, and the BIOG nanoparticles showed in vitro biocompatibility, since they did not interfere in the expression of GFAP by MIO-M1 cells and did not induce detectable modification in the cell phenotype that exert an important role in the maintenance of structure and functions of the retina.

The biocompatibility of BIOG PCL nanofibers was also investigated using the HET-CAM model. The HET-CAM assay is based on the direct application of a sample onto the CAM of hen's egg and the observation of vascular injury or coagulation in response to the intolerance to the sample. These signs could be clearly observed, since the CAM is highly vascularized, as the conjunctiva. However, the CAM has no sensory innervation, which implies the absence of pain in the embryo when irritant substances are evaluated [47]. In this regard, BIOG PCL nanofibers and PCL nanofibers were applied on the CAM surface to verify the possibility of irritation of these samples. However, the nanofibers did not provoke any sign of hyperemia, hemorrhage, and intravasal coagulation, suggesting that they would not damage mucous membranes (especially the eye) [48]. The absence of injury indicated the safety of BIOG PCL nanofibers and their ocular biocompatibility.

\section{Conclusions}

In this study, electrospun PCL nanofibers containing BIOG nanoparticles were synthesized. The low concentration of BIOG nanoparticles $[1 \%(\mathrm{w} / \mathrm{v})]$ into the PCL nanofibers restricted their detection and conclusive characterization by FTIR and DSC. However, the BIOG nanoparticles into polymeric nanofibers were detected by SEM, and they were able to increase the thickness of fibers and change their morphology into a rough surface. Moreover, the BIOG nanoparticles altered the degradation profile of PCL nanofibers due to their bioactive behavior. The presence of BIOG nanoparticles and the protuberances of polymeric nanofibers may have induced the absorption of serum proteins on their surface, resulting in the adhesion and proliferation of ARPE-19 cells due to the establishment of cell-substrate interactions. It was also considered that the 3D network of nanofibers and the porosity allowed the anchorage of RPE cells on the substrates. As a result, the ARPE-19 cells on the BIOG PCL nanofibers formed a confluent monolayer of cells highly connected, thereby, demonstrating the applicability of these nanofibers as temporary supports for cellular therapy aiming at the treatment of AMD. In this regard, considering the possibility of indirect contact of BIOG PCL nanofibers with the neurosensory retina after transplantation to the subretinal space, the biocompatibility of nanofibers was evaluated using MIOM1 cells, which are involved in the retina homeostasis. The glial cells proliferated and showed viability in the presence of BIOG PCL nanofibers, the by-products of the polymer, 
and the BIOG nanoparticles. Additionally, the MIO-M1 cells exhibited functionality due to the expression of GFAP. All these findings associated with the tolerance of the CAM suggested the absence of toxicity and the in vitro biocompatibility of hybridized nanofibers. Finally, it was hypothesized that the BIOG PCL nanofibers could be applied as biodegradable and biocompatible substrates for ARPE-19 cell transplantation.

\section{Competing Interests}

The authors declare they have no competing interests.

\section{Acknowledgments}

The authors wish to thank the UFSJ (Brazil), CNPq/MCT (Brazil), Fapemig (Brazil), and CAPES (Bolsistas da CAPESBrasília/Brazil) for the financial support.

\section{References}

[1] L. Lu, M. J. Yaszemski, and A. G. Mikos, "Retinal pigment epithelium engineering using synthetic biodegradable polymers," Biomaterials, vol. 22, no. 24, pp. 3345-3355, 2001.

[2] G. R. da Silva, S. C. da Armando Jr., J. B. Saliba et al., "Polyurethanes as supports for human retinal pigment epithelium cell growth," International Journal of Artificial Organs, vol. 34, no. 2, pp. 198-209, 2011.

[3] J. Z. Nowak, "Age-related macular degeneration (AMD): pathogenesis and therapy," Pharmacological Reports, vol. 58, no. 3, pp. 353-363, 2006.

[4] E. Ozaki, M. Campbell, A.-S. Kiang, M. Humphries, S. L. Doyle, and P. Humphries, "Inflammation in age-related macular degeneration," in Retinal Degenerative Diseases. Advances in Experimental Medicine and Biology, J. D. Ash, C. Grimm, J. G. Hollyfield, R. E. Anderson, M. M. LaVail, and C. Bowes Rickman, Eds., pp. 229-235, Springer, New York, NY, USA, 2014.

[5] B. G. Short, "Safety evaluation of ocular drug delivery formulations: techniques and practical considerations," Toxicologic Pathology, vol. 36, no. 1, pp. 49-62, 2008.

[6] T. Y. Wong, G. Liew, and P. Mitchell, "Clinical update: new treatments for age-related macular degeneration," The Lancet, vol. 370, no. 9583, pp. 204-206, 2007.

[7] J. D. Du, W. Fong, S. Caliph, and B. J. Boyd, "Lipid-based drug delivery systems in the treatment of wet age-related macular degeneration," Drug Delivery and Translational Research, 2016.

[8] A. Veis and J. Cohen, "Reversible transformation of gelatin to the collagen structure," Nature, vol. 186, no. 4726, pp. 720-721, 1960.

[9] L. V. Del Priore and T. H. Tezd, "Reattachment rate of human retinal pigment epithelium to layers of human Bruch's membrane," Archives of Ophthalmology, vol. 116, no. 3, pp. 335-341, 1998.

[10] B. Lu, D. Zhu, D. Hinton, M. S. Humayun, and Y.-C. Tai, "Meshsupported submicron parylene-C membranes for culturing retinal pigment epithelial cells," Biomedical Microdevices, vol. 14, no. 4, pp. 659-667, 2012.

[11] B. O. Pennington and D. O. Clegg, "Pluripotent stem cell-based therapies in combination with substrate for the treatment of age-related macular degeneration," Journal of Ocular Pharmacology and Therapeutics, vol. 32, no. 5, pp. 261-271, 2016.
[12] V. Kearns, A. Mistry, S. Mason et al., "Plasma polymer coatings to aid retinal pigment epithelial growth for transplantation in the treatment of age related macular degeneration," Journal of Materials Science: Materials in Medicine, vol. 23, no. 8, pp. 20132021, 2012.

[13] P. H. Warnke, M. Alamein, S. Skabo et al., "Primordium of an artificial Bruch's membrane made of nanofibers for engineering of retinal pigment epithelium cell monolayers," Acta Biomaterialia, vol. 9, no. 12, pp. 9414-9422, 2013.

[14] G. C. Ingavle and J. K. Leach, "Advancements in electrospinning of polymeric nanofibrous scaffolds for tissue engineering," Tissue Engineering - Part B: Reviews, vol. 20, no. 4, pp. 277-293, 2014.

[15] P. Xiang, K.-C. Wu, Y. Zhu et al., "A novel Bruch's membranemimetic electrospun substrate scaffold for human retinal pigment epithelium cells," Biomaterials, vol. 35, no. 37, pp. 97779788, 2014.

[16] J. Pelipenko, P. Kocbek, and J. Kristl, "Critical attributes of nanofibers: preparation, drug loading, and tissue regeneration," International Journal of Pharmaceutics, vol. 484, no. 1-2, pp. 5774, 2015.

[17] Y. J. Son, W. J. Kim, and H. S. Yoo, "Therapeutic applications of electrospun nanofibers for drug delivery systems," Archives of Pharmacal Research, vol. 37, no. 1, pp. 69-78, 2014.

[18] A. A. R. De Oliveira, D. A. De Souza, L. L. S. Dias, S. M. De Carvalho, H. S. Mansur, and M. De Magalhães Pereira, "Synthesis, characterization and cytocompatibility of spherical bioactive glass nanoparticles for potential hard tissue engineering applications," Biomedical Materials (Bristol), vol. 8, no. 2, Article ID 025011, 2013.

[19] W. Stöber, A. Fink, and E. Bohn, "Controlled growth of monodisperse silica spheres in the micron size range," Journal of Colloid And Interface Science, vol. 26, no. 1, pp. 62-69, 1968.

[20] C. Jeffrey-Brinker and W. G. Scherer, The Sol-Gel Science: The Physics and Chemistryof Sol-Gel Processing, Academic Press, New York, NY, USA, 1990.

[21] A. A. R. de Oliveira, S. M. de Carvalho, M. de Fátima Leite, R. L. Oréfice, and M. de Magalhães Pereira, "Development of biodegradable polyurethane and bioactive glass nanoparticles scaffolds for bone tissue engineering applications," Journal of Biomedical Materials Research-Part B: Applied Biomaterials, vol. 100, no. 5, pp. 1387-1396, 2012.

[22] G. R. Da Silva, T. H. Lima, R. L. Oréfice et al., "In vitro and in vivo ocular biocompatibility of electrospun poly $(\varepsilon-$ caprolactone) nanofibers," European Journal of Pharmaceutical Sciences, vol. 73, pp. 9-19, 2015.

[23] J. F. De Souza, K. N. Maia, P. S. De Oliveira Patrício et al., "Ocular inserts based on chitosan and brimonidine tartrate: development, characterization and biocompatibility," Journal of Drug Delivery Science and Technology, vol. 32, pp. 21-30, 2016.

[24] H.-M. Lin, Y.-H. Lin, and F.-Y. Hsu, "Preparation and characterization of mesoporous bioactive glass/polycaprolactone nanofibrous matrix for bone tissues engineering," Journal of Materials Science: Materials in Medicine, vol. 23, no. 11, pp. 26192630, 2012.

[25] R. G. Alany, T. Rades, J. Nicoll, I. G. Tucker, and N. M. Davies, "W/O microemulsions for ocular delivery: evaluation of ocular irritation and precorneal retention," Journal of Controlled Release, vol. 111, no. 1-2, pp. 145-152, 2006.

[26] F. A. P. de Sá, S. F. Taveira, G. M. Gelfuso, E. M. Lima, and T. Gratieri, "Liposomal voriconazole (VOR) formulation for 
improved ocular delivery," Colloids and Surfaces B: Biointerfaces, vol. 133, pp. 331-338, 2015.

[27] A. Sene, A. A. Khan, D. Cox et al., "Impaired cholesterol efflux in senescent macrophages promotes age-related macular degeneration," Cell Metabolism, vol. 17, no. 4, pp. 549-561, 2013.

[28] Z. X. Meng, W. Zheng, L. Li, and Y. F. Zheng, "Fabrication and characterization of three-dimensional nanofiber membrance of PCL-MWCNTs by electrospinning," Materials Science and Engineering C, vol. 30, no. 7, pp. 1014-1021, 2010.

[29] P. Wutticharoenmongkol, N. Sanchavanakit, P. Pavasant, and P. Supaphol, "Preparation and characterization of novel bone scaffolds based on electrospun polycaprolactone fibers filled with nanoparticles," Macromolecular Bioscience, vol. 6, no. 1, pp. 70-77, 2006.

[30] T. Osathanon, K. Bespinyowong, M. Arksornnukit, H. Takahashi, and P. Pavasant, "Human osteoblast-like cell spreading and proliferation on Ti-6Al-7Nb surfaces of varying roughness," Journal of Oral Science, vol. 53, no. 1, pp. 23-30, 2011.

[31] M. M. Coleman and J. Zarian, "Fourier-transform infrared studies of polymer blends. II. Poly( $\epsilon$-caprolactone)-poly(vinyl chloride) system," Journal of Polymer Science Part B: Polymer Physics, vol. 17, no. 5, pp. 837-850, 1979.

[32] T. Elzein, M. Nasser-Eddine, C. Delaite, S. Bistac, and P. Dumas, "FTIR study of polycaprolactone chain organization at interfaces," Journal of Colloid and Interface Science, vol. 273, no. 2, pp. 381-387, 2004.

[33] M. Mozafari, F. Moztarzadeh, and M. Tahriri, "Investigation of the physico-chemical reactivity of a mesoporous bioactive $\mathrm{SiO}_{2}-\mathrm{CaO}-\mathrm{P}_{2} \mathrm{O}_{5}$ glass in simulated body fluid," Journal of NonCrystalline Solids, vol. 356, no. 28-30, pp. 1470-1478, 2010.

[34] A. Saboori, M. Rabiee, F. Moztarzadeh, M. Sheikhi, M. Tahriri, and M. Karimi, "Synthesis, characterization and in vitro bioactivity of sol-gel-derived $\mathrm{SiO}_{2}-\mathrm{CaO}-\mathrm{P}_{2} \mathrm{O}_{5}-\mathrm{MgO}$ bioglass," Materials Science and Engineering C, vol. 29, no. 1, pp. 335-340, 2009.

[35] M. H. Aburahma and A. A. Mahmoud, "Biodegradable ocular inserts for sustained delivery of brimonidine tartarate: preparation and in vitro/in vivo evaluation," AAPS PharmSciTech, vol. 12, no. 4, pp. 1335-1347, 2011.

[36] G. Ciardelli, V. Chiono, G. Vozzi et al., "Blends of poly-( $\varepsilon$ caprolactone) and polysaccharides in tissue engineering applications," Biomacromolecules, vol. 6, no. 4, pp. 1961-1976, 2005.

[37] D. Kolbuk, P. Sajkiewicz, P. Denis, and E. Choinska, "Investigations of polycaprolactone/gelatin blends in terms of their miscibility," Bulletin of the Polish Academy of Sciences, vol. 61, no. 3, pp. 629-632, 2013.

[38] Š. Zupančič, S. Sinha-Ray, S. Sinha-Ray, J. Kristl, and A. L. Yarin, "Controlled release of ciprofloxacin from core-shell nanofibers with monolithic or blended core," Molecular Pharmaceutics, vol. 13, no. 4, pp. 1393-1404, 2016.

[39] I. Izquierdo-Barba, D. Arcos, Y. Sakamoto, O. Terasaki, A. López-Noriega, and M. Vallet-Regí, "High-performance mesoporous bioceramics mimicking bone mineralization," Chemistry of Materials, vol. 20, no. 9, pp. 3191-3198, 2008.

[40] K. Rechendorff, M. B. Hovgaard, M. Foss, V. P. Zhdanov, and F. Besenbacher, "Enhancement of protein adsorption induced by surface roughness," Langmuir, vol. 22, no. 26, pp. 10885-10888, 2006.

[41] S. Sharma, S. Mohanty, D. Gupta, M. Jassal, A. K. Agrawal, and R. Tandon, "Cellular response of limbal epithelial cells on electrospun poly- $\varepsilon$-caprolactone nanofibrous scaffolds for ocular surface bioengineering: a preliminary in vitro study," Molecular Vision, vol. 17, pp. 2898-2910, 2011.

[42] G. E. Korte, E. Mrowiec, K. Starer Landzberg, and A. Youssri, "Reorganization of actin microfilaments and microtubules in regenerating retinal pigment epithelium," Experimental Eye Research, vol. 61, no. 2, pp. 189-203, 1995.

[43] A. Bringmann, T. Pannicke, J. Grosche et al., "Müller cells in the healthy and diseased retina," Progress in Retinal and Eye Research, vol. 25, no. 4, pp. 397-424, 2006.

[44] A. Reichenbach and A. Bringmann, "New functions of müller cells," Glia, vol. 61, no. 5, pp. 651-678, 2013.

[45] S.-F. Lin, Y.-X. Mao, B. Li, W. Sun, and S.-B. Tang, "Morphological and immunocytochemical analysis of human retinal glia subtypes in vitro," International Journal of Ophthalmology, vol. 6, no. 5, pp. 559-563, 2013.

[46] A. Bringmann, I. Iandiev, T. Pannicke et al., "Cellular signaling and factors involved in Müller cell gliosis: neuroprotective and detrimental effects," Progress in Retinal and Eye Research, vol. 28, no. 6, pp. 423-451, 2009.

[47] A. L. Savian, D. Rodrigues, J. Weber et al., "Dithranol-loaded lipid core nanocapsules improve the photostability and reduce the in vitro irritation potential of this drug," Materials Science \& Engineering. C, Materials for Biological Applications, vol. 46, pp. 69-76, 2015.

[48] J. Tavaszi and P. Budai, "The use of HET-CAM test in detecting the ocular irritation," Communications in Agricultural and Applied Biological Sciences, vol. 72, no. 2, pp. 137-141, 2007. 

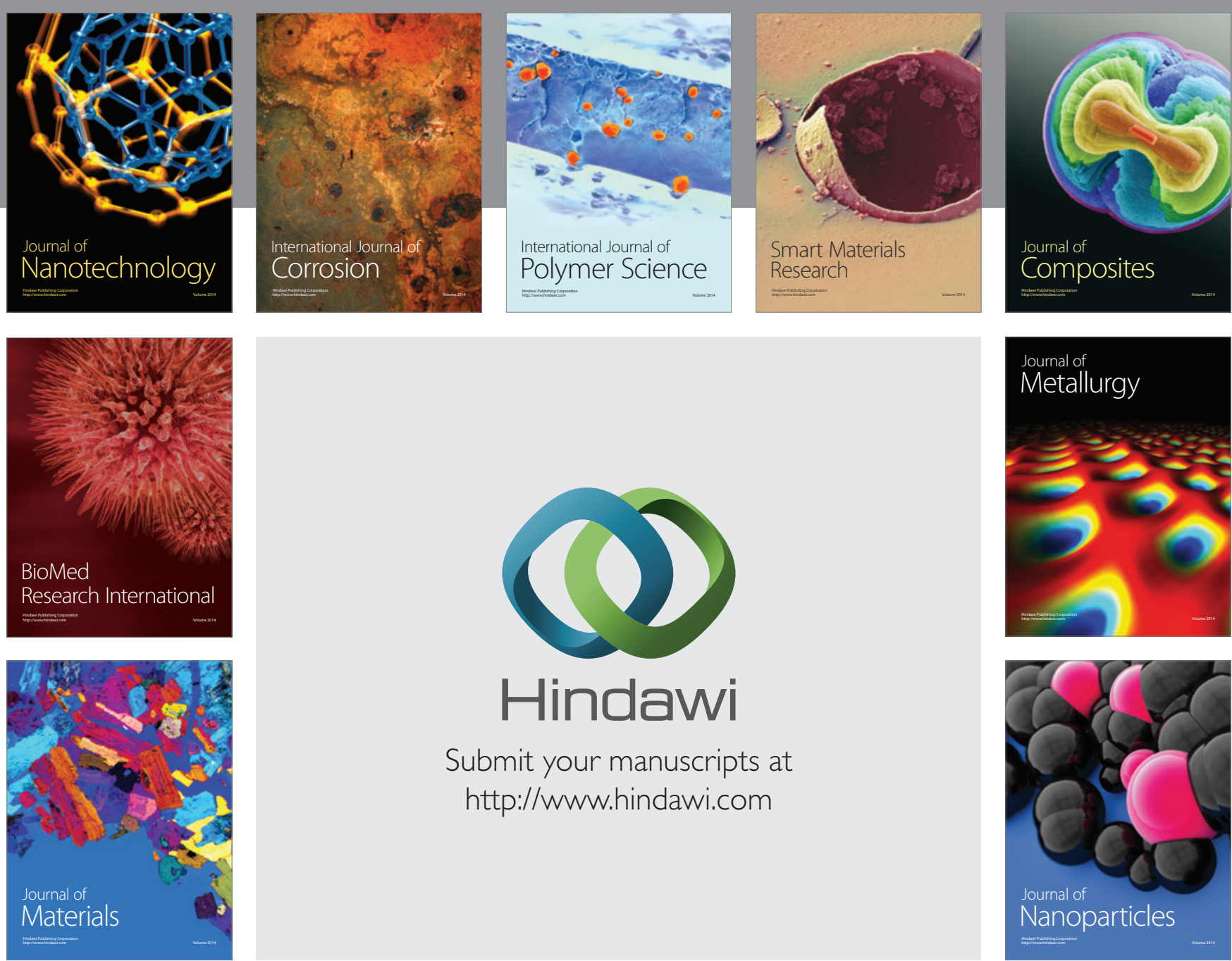

\section{Hindawi}

Submit your manuscripts at

http://www.hindawi.com

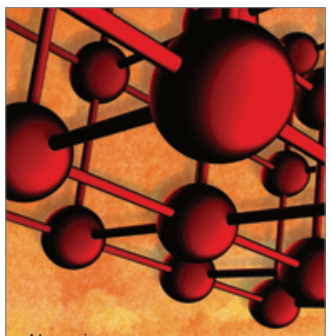

Materials Science and Engineering
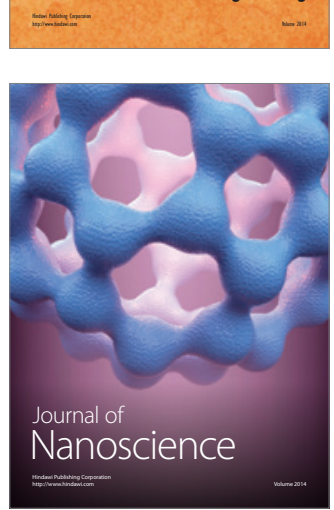
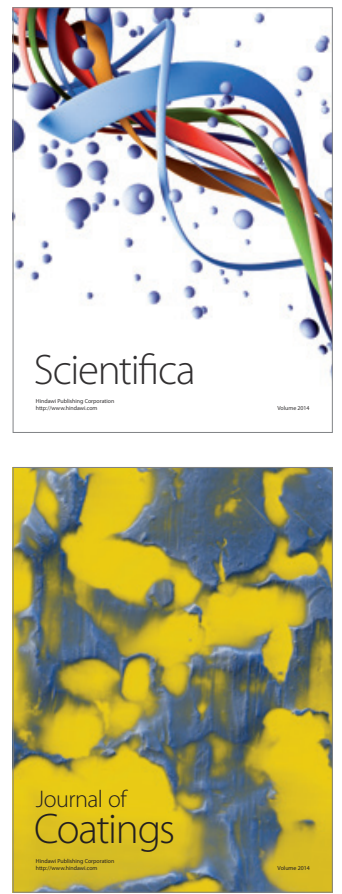
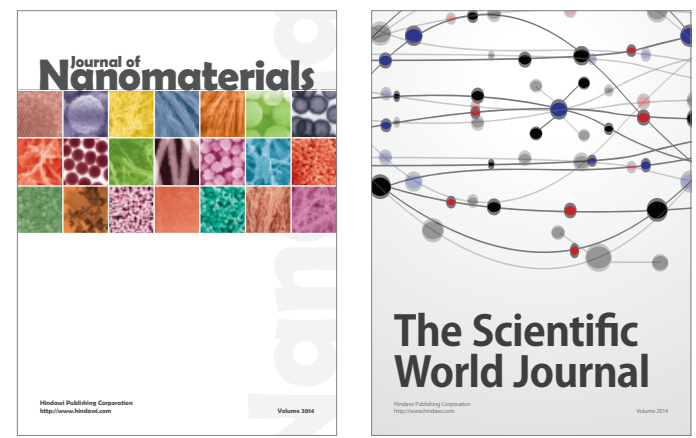

The Scientific World Journal
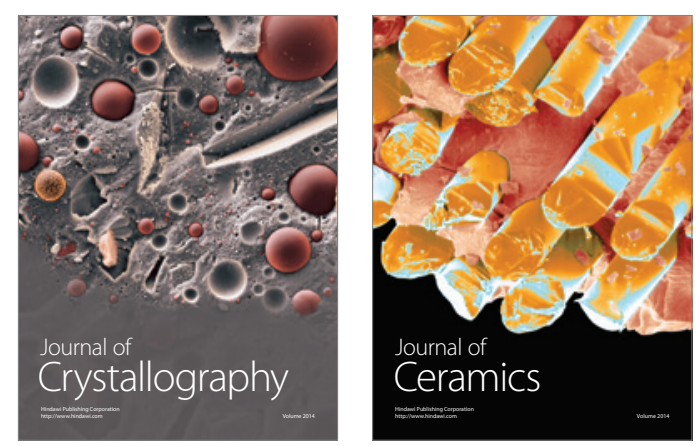
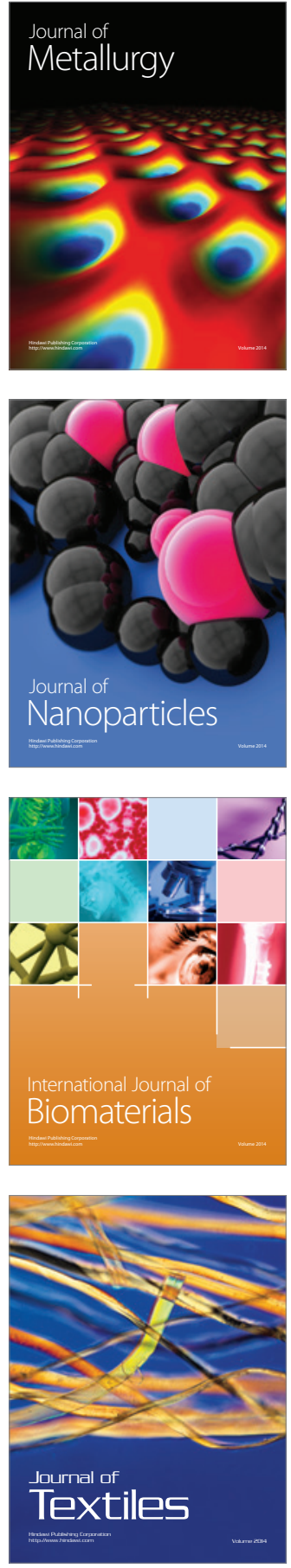